\title{
High Performance Asymmetric Three Corrugation-Pitch-Modulated DFB Lasers Suitable for Stable Single Longitudinal Mode Operation
}

\author{
Qiang Zuo*, Jianyi Zhao, Zhihao Wang, Xin Chen, Wen Liu \\ Wuhan National Laboratory for Optoelectronics, School of Optical and Electronic Information, \\ Huazhong University of Science and Technology, Wuhan 430074, China \\ Email: 2004zuoyou@163.com
}

Received 2013

\begin{abstract}
This paper presents an optimized asymmetric three corrugation-pitch-modulated DFB laser (3CPM-DFB) with extremely high mode selectivity $(\Delta \alpha L=0.97)$ and low flatness $(F=0.009)$, which are two key parameters to indicate the laser's single longitudinal mode(SLM) performance. In threshold analysis, the optimization process based on transfer matrix method is demonstrated to maximize $\Delta \alpha L$ and minimize $F$ simultaneously. In the above-threshold regime, the evolutions of $\Delta \alpha L$ and longitudinal distribution of photon density with injection current are evaluated. More importantly, nanoimprint lithography which was proved an efficient way to fabricate DFB gratings can provide completely same simple fabrication procedure for both $3 \mathrm{CPM}$ grating and conventional uniform grating. So the big practical value of 3CPM-DFB can be expected because of its advanced performance and easy manufacturability.
\end{abstract}

Keywords: Corrugation-Pitch-Modulated; Distributed Feedback Laser; Mode Selectivity; Flatness

\section{Introduction}

With the development requirements of metro and access networks, low cost and high stable single longitudinal mode (SLM) oscillation DFB lasers are desired strongly[1]. The diffraction grating structure is one of the key parts deciding the laser's SLM performance, which usually indicated by two parameters: mode selectivity $(\Delta \alpha L)$ and cavity field flatness factor $(F)$. Actually, to avoid the degradation of SLM operation of conventional quarterly-wavelength shifted (QWS) grating DFB laser induced by spatial hole burning (SHB) effect, there is a long research history of grating optimization design. Mainly they are classified into four categories:(1)gain coupling grating[2]; (2)distributed coupling coefficient (DCC) grating [3]; (3)corrugation pitch modulated (CPM) grating[4]; (4) multiple phase-shift (MPS) grating[5]. Of course, appropriate combination of phase-shift or CPM and DCC grating can give better SLM performance, despite the fact that the difficulties of manufacture increase dramatically[6].

The major drawback of gain coupling grating and DCC grating is fabrication difficulties, making them hard to implement. As far as manufacturing method of diffraction grating is concerned, nanoimprint lithography (NIL), as a promising nano-structure fabrication method with high resolution, high throughput and low cost, was pro- posed to fabricate diffraction gratings of DFB laser recently [7-8]. The characteristics of NIL make it provide completely apparent manufacturing processes to MPS type and CPM type grating with no more complexity. To the authors' knowledge, however, there hasn't been accurate optimization research of multiple CPM grating DFB laser up to now. In this work, an asymmetric three CPM grating is optimized to improve the laser's SLM performance. Results show that 3CPM-DFB can give much bigger $\Delta \alpha L$ and smaller $F$ than 3PS-DFB, namely, more stable SLM performance. In Section 2, the classical transfer matrix method (TMM) is used for threshold analysis and grating structure optimization. Then above threshold analysis of the optimized 3CPM-DFB is investigated in Section 3. The variations of $\Delta \alpha L$ and longitudinal distribution of photon density versus injection current are calculated. Finally, Section 4 summarizes the main conclusions.

\section{3CPM Grating Structure and Optimization}

\subsection{CPM Grating Structure}

Figure 1 schematically shows the 3CPM grating structure under analysis. Both ends of the grating are perfectly anti-reflection coated and the coupling coefficient is uniform along the axis. The unique character of 3CPM grat- 
ing is that there are three Bragg-detuned sections with the grating period bigger than that of the other parts, namely, the basic period. As shown in Figure 1, $r_{1}, r_{2}, r_{3}$ are defined as the center of the three CPM parts respectively and length of the three CPM parts are $L c_{1}, L c_{2}, L c_{3}$. All these parameters are normalized to the total cavity length $L$.

$\Lambda_{j}(j=1,2,3)$ are the grating period of the three CPM parts respectively. $\Lambda_{0}$ is the basic period. Define the relative pitch difference of three CPM parts to basic period as follows:

$$
\Delta \Lambda_{3}=\frac{\Lambda_{j}-\Lambda_{0}}{\Lambda_{0}}, j=1,2,3
$$

Here the center of the second CPM part is limited to the center of the cavity. That means $r_{2}=0.5$. So the rest of parameters need to be optimized are $r_{1}, r_{3}, L c_{1}$, $L c_{2}, L c_{3}, \Delta \Lambda_{1}, \Delta \Lambda_{2}, \Delta \Lambda_{3}$ and normalized coupling coefficient ( $K L)$. Think of the fact that the effect of relative pitch difference to device performance is periodic[4], the varying range of relative pitch difference is limited between 0 and $5 \times 10^{-3} \quad K L$ is limited between 1 and 3 . The constraints of $r_{1}, r_{3}, L c_{1}, L c_{2}, L c_{3}$ are to avoid the overlap of the three CPM parts. The other laser parameters used in this paper are summarized in Table $\mathbf{1 .}$

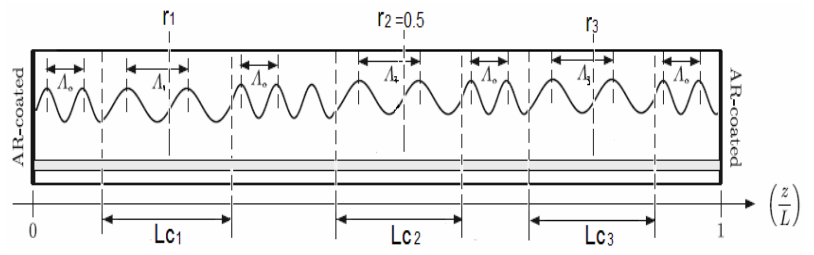

Figure 1. A simplified schematic diagram for the 3CPM-DFB laser structure.

Table 1. Summary of laser parameters.

\begin{tabular}{lc}
\hline \multicolumn{1}{c}{ Laser parameters } & Value \\
\hline Materials parameters & \\
Spontaneous emission rate, A & $2.5 \times 10^{8} \mathrm{~s}^{-1}$ \\
Bimolecular recombination coefficient, B & $1.0 \times 10^{-16} \mathrm{~m}^{3} \cdot \mathrm{s}^{-1}$ \\
Auger recombination coefficient, $\mathrm{C}$ & $3.0 \times 10^{-41} \mathrm{~m}^{6} \cdot \mathrm{s}^{-1}$ \\
Differential gain, $\mathrm{A}_{0}$ & $2.7 \times 10^{-20} \mathrm{~m}^{2}$ \\
Gain curvature, $\mathrm{A}_{1}$ & $1.5 \times 10^{19} \mathrm{~m}^{-3}$ \\
Differential peak wavelength, $\mathrm{A}_{2}$ & $2.7 \times 10^{-32} \mathrm{~m}^{4}$ \\
Internal loss, $\alpha_{\text {loss }}$ & $4.0 \times 10^{3} \mathrm{~m}^{-1}$ \\
Effective index at zero injection, $\mathrm{n}_{0}$ & 3.41351524 \\
Carrier density at transparency, $\mathrm{N}_{0}$ & $1.5 \times 10^{24} \mathrm{~m}^{-3}$ \\
Differential index, dn/dN & $-1.8 \times 10^{-26} \mathrm{~m}^{3}$ \\
Group velocity, vg & $8.1 \times 10^{7} \mathrm{~m}^{-1} \mathrm{~s}^{-1}$ \\
Nonlinear gain coefficient, $\varepsilon$ & $1.5 \times 10^{-23} \mathrm{~m}^{3}$ \\
Structure parameters & \\
Active layer width, w & $1.5 \mu \mathrm{m}$ \\
Active layer thickness, $\mathrm{d}$ & $0.12 \mu \mathrm{m}$ \\
Cavity length, L & $500 \mu \mathrm{m}$ \\
Optical confinement factor, $\Gamma$ & 0.35 \\
Grating period, $\Lambda$ & $227.039 \mathrm{~nm}^{2}$ \\
\hline
\end{tabular}

\subsection{Grating Structure Optimization}

To do the threshold analysis, sophisticated TMM-based laser model is used [5-6]. The cavity is divided into seven concatenated sections to ensure structure parameters in each section are constant. For a given set of structure parameters, the entire lasing mode and their threshold gain can be obtained based on TMM model. Then the mode selectivity and field flatness can be calculated. They are defined by

$$
\Delta \alpha L=\alpha L-\alpha_{t h} L ; F=\frac{1}{L} \int_{0}^{L}[I(z)-\bar{I}]^{2} d z
$$

Where $\alpha_{t h} L$ and $\alpha L$ are the normalized gain of the lasing mode and the main side mode respectively. $I(z)$ is the normalized electric field intensity at position $z$ and $I$ is its average value along the cavity. The set of structure parameters mentioned in section 2.1 are scanned and updated with the similar step by step procedures that are clearly presented in reference [5,6]. In every step, the bigger $\Delta \alpha L$ and smaller $F$ than the previous step can be picked out and the corresponding structure parameters can be updated. Then other parameters are ready to optimized. This process is repeated until no improvements on $\Delta \alpha L$ and $F$ are achieved.

The final results are summarized in Table 2 and compared with that of the 3PS-DFB reported in literature [5] and QWS-DFB. It is clear that the optimized asymmetric 3CPM-DFB have big advantages with much higher $\Delta \alpha L$ and extremely low $F$. And the optimized structure parameters are as follows: $r_{1}=0.135, r_{3}=0.78, L c_{1}=$ $0.26, \quad L c_{2}=0.23, \quad L c_{3}=0.29, \quad K L=1.7$, $\Delta \Lambda_{1}=2.3 \times 10^{-3}, \Delta \Lambda_{2}=3.4 \times 10^{-3}, \Delta \Lambda_{3}=1.4 \times 10^{-3}$, Figure 2 shows the mode distribution of this optimized structure.

Table 2. $\Delta \alpha L, F$ and $\alpha_{t h} L$ for several laser structures.

\begin{tabular}{lccc}
\hline \multicolumn{1}{c}{ Laser Structure } & $\Delta \alpha L$ & $F$ & $\alpha_{t h} L$ \\
\hline Optimized 3CPM-DFB & 0.97 & 0.009 & 1.14 \\
QWS-DFB & 0.73 & 0.3 & 0.7 \\
Optimized 3PS-DFB[5] & 0.78 & 0.01 & 1.18 \\
\hline
\end{tabular}

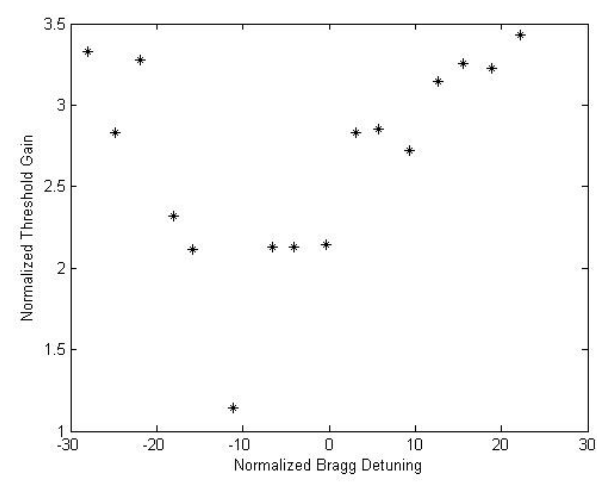

Figure 2. Mode distribution of the optimized structure. 


\section{Above Threshold Analysis}

Even if the threshold analysis presents a good performance of the optimized 3CPM-DFB, an above threshold analysis is essential to assess the effect of SHB on the laser performance with the increasing injection current. In the above threshold regime, the longitudinal inhomogeneities of the photo density, the carrier density and the refractive index have to be considered. The basic model is TMM together with rate equation. The details of lasing mode and side mode analysis are clearly presented in reference [5-6]

Figure 3 shows the longitudinal distribution of the photon density in the optimized asymmetric 3CPM-DFB laser under different biasing currents. Due to the stimulated emission, gradual increase of the photon number in the whole structure can be observed. The meaningful feature is that the difference between the central photon density and the escaping photon densities at the facets is very small. This is beneficial when taking the emitted power into consider.

The mode selectivity of the optimized asymmetric 3CPM-DFB versus biasing current is shown in Figure 4, compared with that of the optimized 3PS-DFB in reference [5] and QWS-DFB. Undoubtedly, the optimized $3 \mathrm{CPM}-\mathrm{DFB}$ is the best and very stable. On the contrary, there are different degrees decreasing of mode selectivity versus current both in 3PS-DFB and QWS-DFB. This represents the optimized 3CPM-DFB is immune to the SHB.

\section{Conclusions}

An asymmetric 3CPM-DFB laser grating structure has been proposed and analyzed in the threshold and above threshold regime. The optimized 3CPM-DFB has extremely high mode selectivity and low field flatness. The

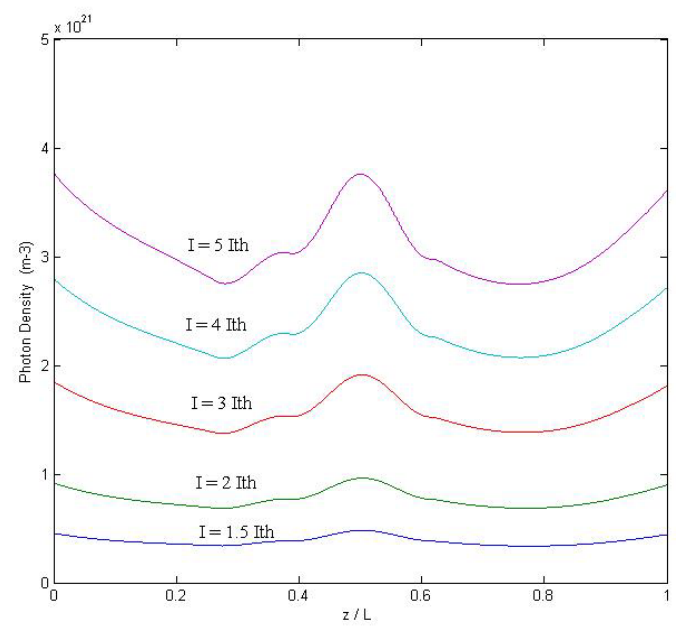

Figure 3. Longitudinal distribution of the photon density in the optimized structure under different biasing currents.

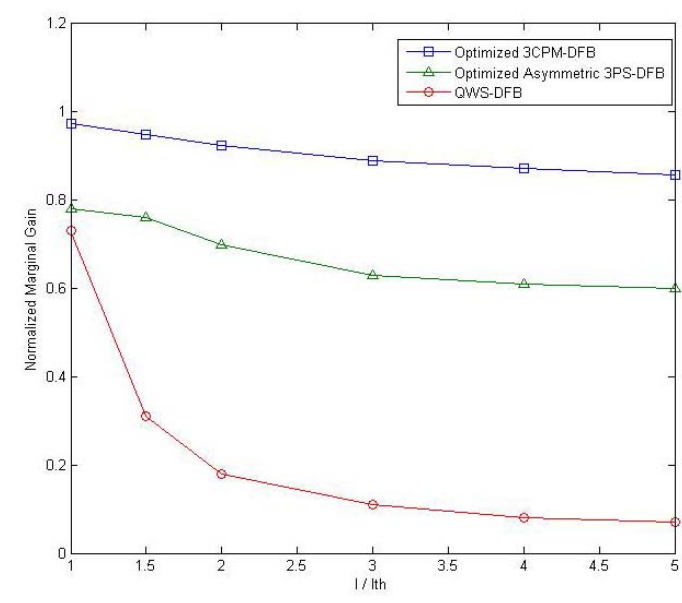

Figure 4. Mode selectivity vs. current injection of the optimized structure, the optimized 3PS-DFB of reference [5] and the QWS-DFB laser structures.

above threshold analysis shows the longitudinal distribution of photon density is still flat enough and mode selectivity is still very high even under $5 I_{t h}$, representing this optimized 3CPM-DFB laser is immune to SHB with high stable single mode operation and very suitable to modern optical communication system.

\section{Acknowledgements}

This work was supported by the Special Project on Development of national key scientific instruments and equipment of China (Grant No. 2011YQ16000205); the National key technology R\&D program of China (Grant No. 2009BAH49B01), and the Chinese National Science and Technology Plan 863 (No 2011AA010304).

\section{REFERENCES}

[1] E. Desurvire, et al. "Science and Technology Challenges in XXIst Century Optical Communications," Comptes Rendus Physique, Vol. 12, No. 4, 2011, pp. 387-416. doi:10.1016/j.crhy.2011.04.009

[2] B. Borchert, et al. " $1.55 \mu \mathrm{m}$ Gain-Coupled Quantum-Well Distributed Feedback Lasers with High Single-Mode Yield and Narrow Linewidth," IEEE Photonics Technology Letters, Vol.3, No. 11, 1991, pp. 955-957.

[3] J. A. P. Morgado, C. A. F. Fernandes, J. B. M. Boavida, "Novel DFB Laser Structure Suitable for Stable Single Longitudinal Mode Operation," Optics \& Laser Technology, Vol. 42, No. 6, 2010, pp. 975-984. doi:10.1016/j.optlastec.2010.01.018

[4] H. Olesen, et al. "Single-Mode Stability of DFB Lasers with Longitudinal Bragg Detuning," IEEE Photonics Technology Letters, Vol. 7, No. 5, 1995, pp. 461-463.

[5] C. A. F. Fernandes, J. A. P. Morgado and J. B. M. Boavida, "Optimisation of an Asymmetric Three Phase-Shift Distributed Feedback Semiconductor Laser" European Physical Journal-applied Physics, Vol. 46, No. 
3, 2009, pp. 30701(1-9). doi:10.1051/epjap/2009068

[6] J. B. M. Boavida, J. A. P. Morgado and C. A. F. Fernandes, "Optimisation of a Corrugation-Pitch-Modulated DFB Laser Structure with Inhomogeneous Coupling Coefficient for Stable Single Longitudinal Mode Operation" European Physical Journal-applied Physics, Vol. 48, No. 3, 2009, pp. 30701 (1-10). doi:10.1051/epjap/2009186

[7] L. Wang, et al., "DFB LDs at DWDM Wavelengths Fabricated by a Novel Nanoimprint Process for Mass Produc- tion and Tolerance Simulation," Microelectronic Engineering, Vol. 93, No. 1, 2012, pp. 43-49, doi:10.1016/j.mee.2011.08.001

[8] M. Yanagisawa, Y. Tsuji, H. Yoshinaga, N. Kono and K. Hiratsuka, "Evaluation of Nanoimprint Lithography as a Fabrication Process of Phase-Shifted Diffraction Gratings of Distributed Feedback Laser Diodes," Journal of Vacuum Science \& Technology, Vol. 27, No. 6, 2009, pp. 2776-2780. doi:10.1116/1.3244631 